\title{
T1G3 bladder cancer, bacillus Calmette-Guerin and radical cystectomy: continued debate
}

\author{
Andrew Brodie ${ }^{1}$, Nadine McCauley ${ }^{1}$, Jo Cresswell $^{2}$, Nikhil Vasdev $^{1,3}$ \\ ${ }^{1}$ Hertfordshire and Bedfordshire Urological Cancer Centre, Department of Urology, Lister Hospital, Stevenage, UK; ${ }^{2}$ Department of Urology, \\ James Cook University Hospital, Middlesbrough, UK; ${ }^{3}$ School of Life and Medical Sciences, University of Hertfordshire, Hertfordshire, UK \\ Correspondence to: Nikhil Vasdev. Consultant Urological Surgeon and Clinical Senior Lecturer, Department of Urology, Lister Hospital, Stevenage, \\ UK. Email: nikhilvasdev@doctors.org.uk. \\ Provenance: This is an invited Editorial commissioned by Section Editor Xiao Li (Department of Urology, Jiangsu Cancer Hospital \& Jiangsu \\ Institute of Cancer Research \& Nanjing Medical University Affiliated Cancer Hospital, Nanjing, China). \\ Comment on: Soria F, Pisano F, Gontero P, et al. Predictors of oncological outcomes in T1G3 patients treated with BCG who undergo radical \\ cystectomy. World J Urol 2018;36:1775-81.
}

Submitted Nov 06, 2018. Accepted for publication Nov 14, 2018.

doi: $10.21037 /$ tau. 2018.11 .07

View this article at: http://dx.doi.org/10.21037/tau.2018.11.07

T1G3 bladder cancer is an unpredictable beast and remains a controversial topic within the urology community. The optimal treatment strategy is a hotly contested subject; in responders, maintenance intravesical instillations of bacillus Calmette-Guerin (BCG) and close endoscopic management offers adequate cancer control with a bladder sparing approach. However, in non-responders it delays and compromises oncological outcomes in a cohort better served by early radical cystectomy (RC). The stakes remain high; left untreated up to $64 \%$ of T1G3 will exhibit disease progression (1) and long term up to $34 \%$ of patients will die of bladder cancer with either treatment option. treatment (2). This delicate balance between over-treating potential BCG responders with a highly morbid surgical procedure compared to undertreating those likely to progress continues to confound urologists and patients. Soria's study on predictors of oncological outcome in BCG treated T1G3 patients undergoing RC published in August's issue of the World fournal of Urology revisits the debate (3).

Soria et al.'s retrospective multicentre study (23 centres) reports on oncologic outcomes and predictors for RC in patients initially treated with BCG for T1G3 (WHO 1973)/ high grade (HG) (WHO 2004) bladder cancer (3). From a 2,451 strong cohort of T1G3/HG patients receiving BCG between 1991 and 2011, 509 (20.8\%) underwent RC (4). The presence of carcinoma in situ (CIS), tumour size $\geq 3 \mathrm{~cm}$, multifocal tumours, absence of muscle in first resection, presence of $\mathrm{T} 1$ in second resection and lack of maintenance
BCG were all significant predictors of RC. The presence of CIS and tumour size $\geq 3 \mathrm{~cm}$ at diagnosis were associated with the worst prognosis and shortest interval to RC.

A total of $226(44 \%)$ patients progressed to muscle invasive disease prior to RC, 111 (22\%) were upstaged after RC and 172 (34\%) patients did not progress. Of the 337 patients that progressed, 153 (45\%) had locally advanced T3/T4 and $72(21 \%)$ had positive lymph nodes $\left(\mathrm{N}_{+}\right)$. The presence of CIS, tumour size $\geq 3 \mathrm{~cm}$ and multiple tumours at diagnosis were all predictors of pathological upstaging to $\mathrm{T} 3 / 4 / \mathrm{N}+$ disease after RC. One hundred and thirteen (22\%) patients died a cancer-related death, $76(67 \%)$ of these exhibited progression prior to RC, 25 (22\%) were upstaged after RC and 12 (11\%) showed no progression. Time to cancer related death was shorter in the 27 (33\%) patients with disease progression. Furthermore, those with recognized progression prior to $\mathrm{RC}$ had a shorter time to cancer death compared to those upstaged on postcystectomy histology. CIS at cystectomy and T3/4 or N+ at cystectomy were associated with shorter disease specific survival.

The authors should be commended on their original approach to describing variables predicting for RC. Most literature on the subject focuses on variables related to disease progression and recurrence. However, in truth there is little fresh insight gained by focusing on risk factors for $\mathrm{RC}$ as these, unsurprisingly, mirror those associated with disease progression and recurrence. Current 2018 EAU 
guidelines on non-muscle invasive bladder cancer (NMIBC) sub-stratify a group of high risk NMIBCs into the 'highestrisk' tumours for progression, based on a variety of multivariate analyses (5). This group includes T1G3/HG with concurrent CIS, multiple and/or large T1G3/HG and/or recurrent T1G3/HG, T1G3/HG with CIS in the prostatic urethra, unusual histology such as micropapillary, plasmacytoid, sarcomatoid and lymphovascular invasion (LVI). For this subgroup, the EAU guidelines recommend early RC should be considered and for those unfit or unwilling BCG treatment should be offered instead. For patients receiving BCG, the EAU recommend consideration of RC in BCG treated patients that progress to muscle invasive disease, continue to have or develop CIS at three months and six months post BCG initiation, those that are intolerant to BCG side effects and those who continue to have G3T1/HG at three months. EAU also recommend upfront RC is considered in patients who recur with G3T1/ HG more than a year post BCG treatment, at which point further BCG or RC should be considered. Given the recommendations for the 'highest risk' subgroup, it is not surprising that patients in the study with CIS and tumours $\geq 3 \mathrm{~cm}$ at diagnosis were more likely to receive $\mathrm{RC}$ earlier, as both are known to increase the risk of disease progression.

The early European Organization for Research and Treatment of Cancer (EORTC) risk calculators based on 2,596 NMIBC cancer patients did not include patients treated with BCG (6). However, the presence of CIS and tumour size $\geq 3 \mathrm{~cm}$ were both shown to worsen prognosis due to increased recurrence rates, additionally CIS increased the risk of progression, corroborating Soria et al.'s findings. To reflect changes in recommendations, especially with regards to the efficacy of maintenance BCG, EORTC published nomograms in 2016 for NMIBC patients receiving one to three years of BCG. They found grade and stage were the most important prognostic factors for progression. For recurrence, the most important prognostic factor was prior recurrence rate and number of tumours (7). However, there is difficulty in comparing this to Soria et al.'s data because the updated EORTC nomogram did not include patients with CIS.

Soria et al.'s study confirms the worsening survival outcomes in patients that progress to muscle invasive disease. Those that progressed prior to RC had worse outcomes compared to those where progression was detected on post cystectomy histology. A significant percentage of patients $(22 \%)$ were upstaged at RC. Similarly, high rates of upstaging at RC have been widely published with figures varying from 21-57\% (8,9). May et al. found that in 210 patients with upstaging at RC, 5-year cancer specific survival reduced from $86 \%$ in the $<$ pT2N0 group to $60 \%$ in the $>$ pT2N0 and worse still in the node positive group at $34 \%$ (9). This is the crux of the argument for early cystectomy as it is widely accepted that delay increases the chance of disease progression and worsens survival outcomes.

There are few shortcomings in the data used in Soria et al.'s study which the authors have acknowledged. These can perhaps be explained by the long period of time over which data was acquired; 1991-2011. Unsurprisingly, urology practice and recommendations have developed and adapted over this 20-year period. One fundamental issue revolves around BCG treatment. Only 142 of the 509 (28\%) patients included in the study received maintenance BCG. Bohle et al.'s meta-analysis recommends that at least 1 year of BCG be given in a maintenance schedule (10) and current EAU guidelines go a step further stating 3-year maintenance is the most effective (5). Thirty years ago, maintenance BCG was not the 'norm' and practices have changed significantly since then. This makes it difficult to draw firm conclusions on the oncological outcomes of BCG treatment in high risk tumours if only $28 \%$ of the patients received what is currently recommended as the optimal BCG schedule. This factor may make the study less generalizable to current practice. Similar criticism can be levelled at the rates of second resection. EAU guidelines recommend all $\mathrm{T} 1$ tumours have a second resection as well as those without muscle in the first resection (5). The largest meta-analysis including 3,566 patients found significant residual tumour, up to $50 \%$, and significant upstaging, $10 \%$, at second resection in $\mathrm{T} 1$ patients (11). Furthermore, it has been shown that residual T1 disease at re-resection is a bad prognostic sign for recurrence and progression and may influence the decision for early $\mathrm{RC}$ (12). The authors appreciate this limitation as they inform us in their conclusion that only $258(51 \%)$ of the patients underwent re-resection. Similarly, we do not know the rates of muscle inclusion in the study data and if we combine this with the low re-resection rates there is serious concern regarding incomplete resection of tumour leading to significant under-staging in this study.

There is little to no mention of neoadjuvant chemotherapy (NAC) or cystectomy technique in Soria et al.'s study. In fairness, there is scant guidance on the role of NAC in patients with T1G3 disease undergoing $\mathrm{RC}$ and it is not recommended by the current guidance. 
There is also limited guidance to suggest whether NAC is beneficial in those that progress to muscle invasive disease prior to RC. However, given cisplatin-based combination NAC has been shown to improve overall 5 -year survival by $8 \%$ in those with muscle invasive disease (13), it is an important variable to consider. Indeed, it may have had a bearing on the survival outcomes described in the study. With regards to cystectomy technique, it can be assumed that the majority were performed by the open approach. From 2008 onwards, the role of minimally invasive robotic techniques has increased due to emerging technology and surgeon experience. Analysis of the US national cancer database reveals that $34.1 \%$ of all cystectomies in 2013 were performed by the minimally invasive approach (14). Studies to date have failed to attribute an oncological advantage to open or minimally invasive techniques, however, this may change with time and for example, facilitate the timely administration of adjuvant chemotherapy with improved outcomes.

Increased interest in the biochemical markers of BCG response, mirrors the growing understanding of the molecular and immunological basis of bladder cancer and BCG response. Fluorescence in situ hybridization (FISH) represents an old technology with a new application. It was originally developed for cancer detection but has since been studied as a marker for predicting BCG failure. A positive FISH result at 3 months correlates with $46 \%$ risk of tumour recurrence compared to an $8 \%$ risk with a FISH negative result $(\mathrm{P}<0.001)(15)$. Overexpression of $\mathrm{p} 53$, a cell cycle regulator, is well investigated and has been associated with disease progression (16). Multiple other molecular markers have been suggested including surviving, Ki-67, ezrin and bcl-2 to name a few. Many of these studies suffer from a lack of standardised measurement and diagnostic cut-offs (17). Further proposed biomarkers include revisiting urinary cytokines with new technologies, measurement of clinical response through surrogates such as skin reactivity in antituberculin reaction and leukocyturia, as well as the genomic sequencing of BCG responders and non-responders. It remains to be seen whether these new technologies will translate into clinical practice.

In conclusion, Soria's paper re-ignites the longstanding debate regarding early cystectomy for T1G3 disease, and confirms our existing understanding of prognostic factors. Whilst they have provided an interesting perspective by investigating the risk factors for $\mathrm{RC}$, the clinical benefit is not convincing, especially considering how explicit current EAU guidelines are in their recommendation for RC in the 'highest risk' subcategory. Furthermore, there are question marks over the clinical applicability of their data, many of the patients received treatment almost thirty years ago, at a time when maintenance BCG and re-resection were not recommended. Looking forward, future well designed randomised prospective trials comparing BCG and early RC will be invaluable, albeit difficult to facilitate. Perhaps the answer will be found in emerging technologies. As our understanding of the molecular and immunological basis of BCG response improves, biomarkers may be able to predict those destined to fail BCG treatment, surpassing our current clinicopathological predictors. Armed with this information, urologists will be able to offer sensible advice to their patients when faced with the dilemma of highly morbid cystectomy versus the potential ineffectiveness of BCG in high risk NMBIC.

\section{Acknowledgements}

None.

\section{Footnote}

Conflicts of Interest: The authors have no conflicts of interest to declare.

\section{References}

1. Nieder AM, Brausi M, Lamm D, et al. Management of stage T1 tumors of the bladder: International Consensus Panel. Urology 2005;66:108-25.

2. Kulkarni GS, Hakenberg OW, Gschwend JE, et al. An updated critical analysis of the treatment strategy for newly diagnosed high-grade T1 (previously T1G3) bladder cancer. Eur Urol 2010;57:60-70.

3. Soria F, Pisano F, Gontero P, et al. Predictors of oncological outcomes in T1G3 patients treated with BCG who undergo radical cystectomy. World J Urol 2018;36:1775-81.

4. Gontero P, Sylvester R, Pisano F, et al. Prognostic factors and risk groups in T1G3 non-muscle-invasive bladder cancer patients initially treated with Bacillus CalmetteGuérin: results of a retrospective multicenter study of 2451 patients. Eur Urol 2015;67:74-82.

5. Babjuk $M$, Böhle A, Burger $M$, et al. EAU Guidelines on Non-Muscle-invasive Urothelial Carcinoma of the Bladder: Update 2016. Eur Urol 2017;71:447-61.

6. Sylvester RJ, van der Meijden AP, Oosterlinck W, et 
al. Predicting recurrence and progression in individual patients with stage Ta T1 bladder cancer using EORTC risk tables: a combined analysis of 2596 patients from seven EORTC trials. Eur Urol 2006;49:466-5; discussion 475-7.

7. Cambier S, Sylvester RJ, Collette L, et al. EORTC Nomograms and Risk Groups for Predicting Recurrence, Progression, and Disease-specific and Overall Survival in Non-Muscle-invasive Stage Ta-T1 Urothelial Bladder Cancer Patients Treated with 1-3 Years of Maintenance Bacillus Calmette-Guérin. Eur Urol 2016;69:60-9.

8. Svatek RS, Shariat SF, Novara G, et al. Discrepancy between clinical and pathological stage: external validation of the impact on prognosis in an international radical cystectomy cohort. BJU Int 2011;107:898-904.

9. May M, Bastian PJ, Brookman-May S, et al. Pathological upstaging detected in radical cystectomy procedures is associated with a significantly worse tumour-specific survival rate for patients with clinical T1 urothelial carcinoma of the urinary bladder. Scand J Urol Nephrol 2011;45:251-7.

10. Böhle A, Bock PR. Intravesical bacille Calmette-Guérin versus mitomycin $\mathrm{C}$ in superficial bladder cancer: formal meta-analysis of comparative studies on tumor progression. Urology 2004;63:682-6; discussion 686-7.

11. Naselli A, Hurle R, Paparella S, et al. Role of Restaging Transurethral Resection for T1 Non-muscle invasive Bladder Cancer: A Systematic Review and Meta-analysis.

Cite this article as: Brodie A, McCauley N, Cresswell J, Vasdev N. T1G3 bladder cancer, bacillus Calmette-Guerin and radical cystectomy: continued debate. Transl Androl Urol 2018;7(Suppl 6):S692-S695. doi: 10.21037/tau.2018.11.07
Eur Urol Focus 2018;4:558-67.

12. Palou J, Pisano F, Sylvester R, et al. Recurrence, progression and cancer-specific mortality according to stage at re-TUR in T1G3 bladder cancer patients treated with BCG: not as bad as previously thought. World J Urol 2018;36:1621-7.

13. Yin M, Joshi M, Meijer RP, et al. Neoadjuvant Chemotherapy for Muscle-Invasive Bladder Cancer: A Systematic Review and Two-Step Meta-Analysis. Oncologist 2016;21:708-15.

14. Bachman AG, Parker AA, Shaw MD, et al. Minimally Invasive Versus Open Approach for Cystectomy: Trends in the Utilization and Demographic or Clinical Predictors Using the National Cancer Database. Urology 2017;103:99-105.

15. Kamat AM, Willis DL, Dickstein RJ, et al. Novel fluorescence in situ hybridization-based definition of bacille Calmette-Guérin (BCG) failure for use in enhancing recruitment into clinical trials of intravesical therapies. BJU Int 2016;117:754-60.

16. Ovesen H, Horn T, Steven K. Long-term efficacy of intravesical bacillus Calmette-Guerin for carcinoma in situ: relationship of progression to histological response and p53 nuclear accumulation. J Urol 1997;157:1655-9.

17. Kamat AM, Li R, O'Donnell MA, et al. Predicting Response to Intravesical Bacillus Calmette-Guérin Immunotherapy: Are We There Yet? A Systematic Review. Eur Urol 2018;73:738-48. 